\section{ACADESINE INHIBITS NEUTROPHIL CD11B UP-REGULATION IN VITRO AND DURING IN VIVO CARDIOPULMONARY BYPASS}

From the Departments of Laboratory Medicine ${ }^{c}$ and Anesthesiology, ${ }^{a}$ Yale University School of Medicine and Yale-New Haven Hospital, and the Multicenter Study of Perioperative Ischemia (McSPI) Research Group, ${ }^{b}$ New Haven, Conn.

Supported by National Institutes of Health grant HL47193 (B.R.S.) and Gensia Pharmaceuticals. Christine Rinder is a recipient of an American Heart Association Clinician-Scientist Award, and Brian Smith is a Scholar of the Leukemia Society of America.

Received for publication Feb. 10, 1994.

Accepted for publication July 12, 1994.

Address for reprints: Christine S. Rinder, MD, Department of Anesthesiology, Yale University School of Medicine, P.O. Box 208035, New Haven, CT 06520-8035.

Copyright (C) 1995 by Mosby-Year Book, Inc. $0022-5223 / 95 \$ 3.00+0 \quad \mathbf{1 2 / 1 / 5 9 1 6 5}$
Granulocyte adhesion to ischemic tissue, mediated in large part by $\beta_{2}$ integrin receptors, is important in the pathophysiology of reperfusion injury. Acadesine, a drug that modulates adenosine levels in ischemic tissue, has been shown to reduce reperfusion injury in animal models of ischemia. The purpose of this study was to measure changes in granulocyte CD11b/CD18 in an in vitro assay and in an in vivo trial of acadesine administered during cardiopulmonary bypass to determine whether this agent might modulate up-regulation of this adhesion receptor. In vitro, whole blood was incubated with acadesine or control diluent, stimulated with $\boldsymbol{N}$-formyl-methionyl-leucylphenylalanine, and granulocyte CD11b measured. Acadesine significantly $(p<0.01$ ) inhibited $N$-formyl-methionyl-leucyl-phenylalanine-induced granulocyte CD11b up-regulation by a mean of $61 \%$. In similar experiments, adenosine also inhibited $N$-formylmethionyl-leucyl-phenylalanine-induced granulocyte CD11b up-regulation $(p<0.01$ ). In vivo, 34 patients at our institution participating in a multicenter trial of acadesine during cardiopulmonary bypass were randomized to placebo, low-dose, or high-dose acadesine infusion perioperatively. Combining low- and high-dose treatment groups, there was significant ( $p=0.05$ ) inhibition of granulocyte CD11b up-regulation in patients receiving acadesine; granulocyte CD11b expression in the acadesine group peaked at 2.8 times baseline versus 4.3 for placebo. By contrast, monocyte CD11b up-regulation (peaking after cardiopulmonary bypass at 3 times baseline) was not affected by acadesine. Acadesine and adenosine inhibit up-regulation of granulocyte CD11b in vitro, and acadesine is capable of a similar inhibition during in vivo cardiopulmonary bypass. This inhibition may contribute to the ability of these agents to decrease in vivo reperfusion injury. (J Thorac Cardiovasc SuRg 1995;109:448-56)

Joseph P. Mathew, MD, ${ }^{\text {a, b }}$ Christine S. Rinder, MD, ${ }^{\mathrm{a}, \mathrm{c}}$ Jayne B. Tracey, BS, Laura A. Auszura, BS, ${ }^{\mathrm{c}}$ Theresa O'Connor, PhD, ${ }^{a}$ Elizabeth Davis, LPN, ${ }^{\mathrm{b}}$ and Brian R. Smith, MD, ${ }^{c}$ New Haven, Conn.
A cadesine (5 amino-4 imidazole carboxamide riboside) is the first of a new group of drugs classified as adenosine-regulating agents whose effects are thought to be mediated by increases in endogenous adenosine levels in ischemic myocardium. ${ }^{1}$ In preclinical studies, acadesine has been shown to inhibit platelet aggregation, ${ }^{2}$ improve postischemic ventricular contractile function, ${ }^{3,4}$ inhibit neutrophil (PMN) adherence to endothelium, ${ }^{5} \mathrm{de}$ crease PMN accumulation in ischemic myocardium, ${ }^{6}$ and decrease free radical- and oxidant-induced cardiac damage. ${ }^{7}$ Mechanisms for acadesine's inhibition of PMN adhesion and respiratory burst, however, have not been elucidated. CD11a/CD18 and CD11b/CD18 are members of the $\beta_{2}$ integrin family of adhesion receptors. CD11b/CD18 in particular permits PMN adhesion to and diapedesis through endothelial cells. ${ }^{8-10}$ Adhesion of PMN via CD11b/ CD18 has been shown to be necessary for secretion of hydrogen peroxide in response to $N$-formylmethionyl-leucyl-phenylalanine (fMLP) or platelet- activating factor. ${ }^{11,12}$ We therefore hypothesized that acadesine-mediated inhibition of up-regulation of $\mathrm{CD} 11 \mathrm{~b} / \mathrm{CD} 18 \mathrm{might}$ be involved in both the inhibition of PMN accumulation and the suppression of oxidative burst observed in the aforementioned studies.

We have previously measured changes in leukocyte and platelet adhesion receptors occurring during cardiopulmonary bypass (CPB). Specifically, we examined changes in the leukocyte adhesion receptor CD11b and demonstrated that PMN and monocyte CD11b were up-regulated threefold to fourfold by the end of CPB. ${ }^{13}$ Similarly, we examined platelet $\mathrm{P}$-selectin, a receptor that is expressed as a consequence of $\alpha$-granule release and that binds activated platelets to leukocytes, and demonstrated both increased platelet surface expression of P-selectin induced by $\mathrm{CPB}^{14}$ and increased platelet-leukocyte conjugate formation during $\mathrm{CPB} .{ }^{13} \mathrm{We}$ and others have also demonstrated changes in the platelet adhesion receptors glycoprotein (GP)Ib and GPIIb/ 
IIIa but not GPIV during CPB. ${ }^{15-19}$ For the current study we used an in vitro whole blood assay to measure changes in PMN CD11b occurring in response to $\mathrm{N}$-formyl-methionyl-leucyl-phenylalanine after incubation with acadesine, adenosine, or control diluent. In addition, in an in vivo study of acadesine during $\mathrm{CPB}$, using techniques described previously, ${ }^{13-15}$ we sought to determine whether acadesine affects either in vivo CPB-induced upregulation of leukocyte adhesion receptors (PMN/ monocyte CD11b and/or CD11a) or platelet adhesion receptors (expression of P-selectin, plateletleukocyte adhesion, and platelet GPIb).

\section{Materials and methods}

Materials. For in vitro studies, acadesine, adenosine, and fMLP were obtained from Sigma Chemical Co. (St. Louis, Mo.); diluents for these materials were phosphatebuffered saline solution for acadesine and adenosine and dimethyl sulfoxide for fMLP. Controls for all reagents in each experiment consisted of the appropriate final concentration of diluent without agonist. For in vivo studies, acadesine was supplied by Gensia Pharmaceuticals (San Diego, Calif.) as part of its multicenter phase II and III trials.

Antibodies. All monoclonal antibodies were used as purified whole immunoglobulin G. All experiments included irrelevant isotype-specific monoclonal antibodies as negative controls. The monoclonal antibody $1 \mathrm{E} 3^{20}$ (gift of Dr. K. Ault, MCRI, So. Portland, Me.) is specific for P-selectin. $\mathrm{P}^{21}$ and $\mathrm{SZ2} 2^{22}$ (AMAC, Inc., Westbrook, Me.) recognize platelet GPIIb/IIIa and GPIb, respectively. Anti-CD45 (HLE, Becton-Dickinson Immunocytometry Systems, San Jose, Calif.) is directed against an antigen present on PMNs, monocytes, and lymphocytes but neither erythroid cells nor platelets. ${ }^{23}$ The monoclonal antibodies D12 ${ }^{24}$ (Leu-15, Becton-Dickinson) and RR3.1 (gift of Dr. R. Rothlein, Boehringer-Ingelheim, Ridgefield, Conn.) recognize leukocyte CD11b and CD11a, respectively.

Fluorescence labeling. As previously detailed, all in vitro and in vivo whole blood samples were fixed for 60 minutes at $4^{\circ} \mathrm{C}$, then washed in Tyrode-HEPES buffer. ${ }^{25}$ Samples from in vitro experiments were studied for PMN CD11b only. Patient samples were divided into three aliquots for study: one for the platelet receptors GPIb and P-selectin, one for the leukocyte adhesion receptors CD11a and CD11b, and one for the percentage of leukocyte-platelet conjugates.

Leukocyte surface CD11a and CD11b were measured by incubating samples with saturating concentrations of fluorescein isothiocyanate (FITC)-anti-CD45 and either biotinylated anti-CD11a or phycoerythrin-anti-CD11b. The samples incubated with anti-CD11a were washed and incubated with saturating concentrations of phycoerythrinstreptavidin (Becton-Dickinson). All samples were then washed and resuspended in Tyrode-HEPES buffer for fluorescence-activated cell sorter (FACS) analysis.
For determination of the surface density of platelet GPIb, $100 \mu$ l of sample was incubated with saturating concentrations of FITC-anti-GPIb, washed, and resuspended in Tyrode-HEPES buffer for flow cytometric analysis as previously described. ${ }^{15}$ To measure the percentage of circulating platelets expressing P-selectin, we incubated a separate sample with FITC-anti-GPIIb/IIIa and biotinylated anti-P-selectin, ${ }^{14}$ washed, and incubated with saturating concentrations of phycoerythrin-streptavidin, washed, and resuspended in Tyrode-HEPES for FACS analysis.

For the percentage of leukocyte-platelet conjugates, the third sample was incubated with FITC-anti-CD45 and biotinylated anti-GPIIb/IIIa, washed, and resuspended in Tyrode-HEPES buffer. Phycoerythrin-streptavidin labeling and preparation for FACS analysis were performed as described earlier.

Flow cytometry. Samples were analyzed on an FACScan flow cytometer (Becton-Dickinson, Mountain View, Calif.) with data stored in list mode files. The determination of the surface density of GPIb and the percentage of platelets expressing P-selectin was carried out as previously described. ${ }^{14,15,26,27}$ The determination of GPIb density always incorporated a single platelet gate using forward scatter to ensure that measurements of GPIb were not biased by microaggregate or microparticle formation. Leukocyte surface CD11a and CD11b were measured by appropriate forward- versus side-scatter gates combined with CD45 expression to distinguish PMNs, monocytes, and lymphocytes. ${ }^{25}$ Quantification of leukocyte-platelet conjugates was based on events that simultaneously fluoresced for both CD45 and GPIIb/IIIa as previously described. ${ }^{13,25}$ As noted earlier, isotype- and fluorochrome-matched control monoclonal antibodies were used in each experiment to determine "nonspecific" background fluorescence.

Leukocyte activation in vitro. All in vitro experiments were conducted in whole blood to minimize CD11b up-regulation associated with leukocyte purification. ${ }^{28}$ Specifically, heparinized whole blood (final heparin concentration 14 units $/ \mathrm{ml}$ ) was incubated with acadesine, adenosine, or diluent for 15 minutes at $37^{\circ} \mathrm{C}$; the doses of acadesine and adenosine used have previously been shown to inhibit other functional changes in PMNs in in vitro experiments. ${ }^{6,29}$ Samples were then stimulated with fMLP for an additional 15 minutes at $37^{\circ} \mathrm{C}$; preliminary experiments demonstrated maximal CD11b expression at this point after addition of fMLP. Samples were then removed directly into $1 \%$ paraformaldehyde and examined for the surface expression of PMN CD11b.

In vivo studies: Patient selection. Studies were performed on patients at Yale University who were enrolled in phase II and phase III of multicenter trials of the myocardial protective effects of acadesine (conducted by the Multicenter Study of Perioperative Ischemia Research Group); the clinical results of phase II have been previously reported. ${ }^{30,31}$ The current study was conducted as part of our ongoing investigation of adhesion receptors during CPB and used only patients studied at Yale University. After institutional approval by the Human Investigation Committee of the Yale University School of Medicine and informed consent, 34 consecutive patients 
undergoing elective coronary artery bypass grafting requiring CPB were studied. Patients excluded from the multicenter study included those with hemodynamically significant valvular disease, patients undergoing other procedures concurrent with the bypass operation, patients with recent or evolving myocardial infarction within 7 days before the operation, patients in cardiogenic shock, and patients with a history of uric acid nephropathy, hepatic insufficiency, or renal insufficiency. Pharmacologic exclusion criteria included the use of adenosine or pentoxifylline within 12 hours of the operation, theophylline and nicotinic acid within 24 hours of the operation, and dipyridamole within 48 hours of the operation. One additional patient was excluded from analysis in the present study because of a hematologic malignancy.

Study protocol. The 34 patients were randomized in double-blind fashion into a placebo group $(n=11)$, a group receiving low-dose acadesine $(0.05 \mathrm{mg} / \mathrm{kg}$ per minute intravenously and $5 \mu \mathrm{g} / \mathrm{ml}$ in cardioplegic solution, $n=11)$, and a group receiving high-dose acadesine $(0.1$ $\mathrm{mg} / \mathrm{kg}$ per minute intravenously and $5 \mu \mathrm{g} / \mathrm{ml}$ in cardioplegic solution, $n=12$ ). Acadesine or placebo was infused continuously beginning 15 minutes before induction of anesthesia and for 7 hours thereafter. Anesthesia was induced and maintained with fentanyl and midazolam, both by bolus followed by infusion, and thiopental up to 7 $\mathrm{mg} / \mathrm{kg}$. Blood pressure and heart rate were maintained within $20 \%$ of baseline values. CPB was conducted with a COBE CML membrane oxygenator (Cobe Laboratories, Inc., Denver, Colo.) and moderate systemic hypothermia $\left(28^{\circ} \mathrm{C}\right)$. Whole blood samples $(200 \mu \mathrm{l})$ were taken from the radial artery catheter and immediately fixed in paraformaldehyde in phosphate-buffered saline solution (1\% final concentration), as previously described. ${ }^{32}$ Samples were taken at the following times: before the start of acadesine infusion, at termination of CPB (before protamine), and 18 hours after CPB. Patient samples were studied for (1) leukocyte surface expression of CD11a and CD11b, (2) platelet activation (P-selectin expression), (3) platelet surface density of GPIb, and (4) percentage of leukocyte-platelet conjugates. CD11a and GPIb were studied only after initiation of phase III of the clinical trial, reducing the number of patients studied to 22 for these receptors. An additional whole blood sample $(1 \mathrm{ml})$ was drawn into ethylenediaminetetraacetic acid $(5 \mathrm{mmol} / \mathrm{L}$ final concentration) at each of the aforementioned times and the total leukocyte count and differential were measured in all 34 patients with an automated Coulter STKS counter (Coulter Electronics, Hialeah, Fla.).

Statistical analysis. Data are expressed as mean \pm standard error of the mean (SEM). Measurements that compared the surface density of a receptor in serial samples on the same patient (e.g., CD11a, CD11b, GPIb) were normalized to the starting value. Measurements that looked at the percentage of positive cells, that is, those which bound monoclonal antibodies above background levels (P-selectin, platelet-PMN, and platelet-monocyte conjugates), were expressed as a percentage of the total number of cells tested per sample. In vitro and demographic data were analyzed by the $t$ test for independent samples to determine whether there was an effect of the drug and by analysis of variance to identify any dose- dependency. For in vivo data, groups were compared by two-factor repeated-measures analysis of variance, where group and time were the factors, with repeats on the time factor. A value of $p \leq 0.05$ was considered statistically significant.

\section{Results}

In vitro leukocyte activation. Samples of whole blood incubated with acadesine or diluent were stimulated with four doses of FMLP and examined for changes in PMN CD11b. In diluent-treated samples, fMLP resulted in a PMN CD11b increase of 1.5-, 1.6-, 1.7-, and 1.8-fold at doses of 5, 10, 50, and $100 \mathrm{nmol} / \mathrm{L}$ respectively. As shown in Fig. $1, A$, acadesine preincubation produced a significant inhibition $(p<0.01)$ of the upregulation in PMN CD11b induced by fMLP as compared with diluenttreated cells (acadesine doses: $1 \mathrm{mmol} / \mathrm{L}, 500$ $\mu \mathrm{mol} / \mathrm{L}$, and $100 \mu \mathrm{mol} / \mathrm{L}$ ), and this effect was doserelated $(p<0.05)$. By examining the fluorescence histograms of CD11b binding, we found that the inhibition of CD11b was uniformly distributed among all PMNs; that is, no PMN subset was disproportionately affected by acadesine.

Whole blood samples were similarly incubated with adenosine or diluent and stimulated with three doses of fMLP as shown in Fig. 1, B. Like acadesine, adenosine demonstrated significant $(p<0.01)$ inhibition of PMN CD11b up-regulation in response to fMLP; this effect was dose-related $(p<0.05)$ and, as with acadesine, affected the entire PMN population.

\section{In vivo studies}

Demographics. Twenty-eight men and six women were studied, averaging 62 years of age. There was no difference between placebo, low-dose, and highdose acadesine groups in either the sex or age distribution. Average CPB time in the placebo group was $87 \pm 10$ minutes (mean \pm SEM), which was significantly shorter $(p=0.02)$ than that of the low-dose acadesine group (117 \pm 6 minutes) and not different from that of the high-dose acadesine group (107 \pm 12 minutes). Similarly, the crossclamp time was significantly shorter $(p=0.05)$ in the placebo group ( $49 \pm 5$ minutes) than in the low-dose acadesine group (63 \pm 4 minutes) but not the highdose acadesine group ( $59 \pm 7$ minutes). When both acadesine treatment groups were combined, the total bypass time was still significantly longer than placebo $(p=0.05)$, but crossclamp time was not significantly longer than placebo $(p=0.08)$.

Monocyte and PMN activation. In placebo-treated patients, surface expression of CD11b on monocytes increased significantly $(p<0.01)$ during and after 


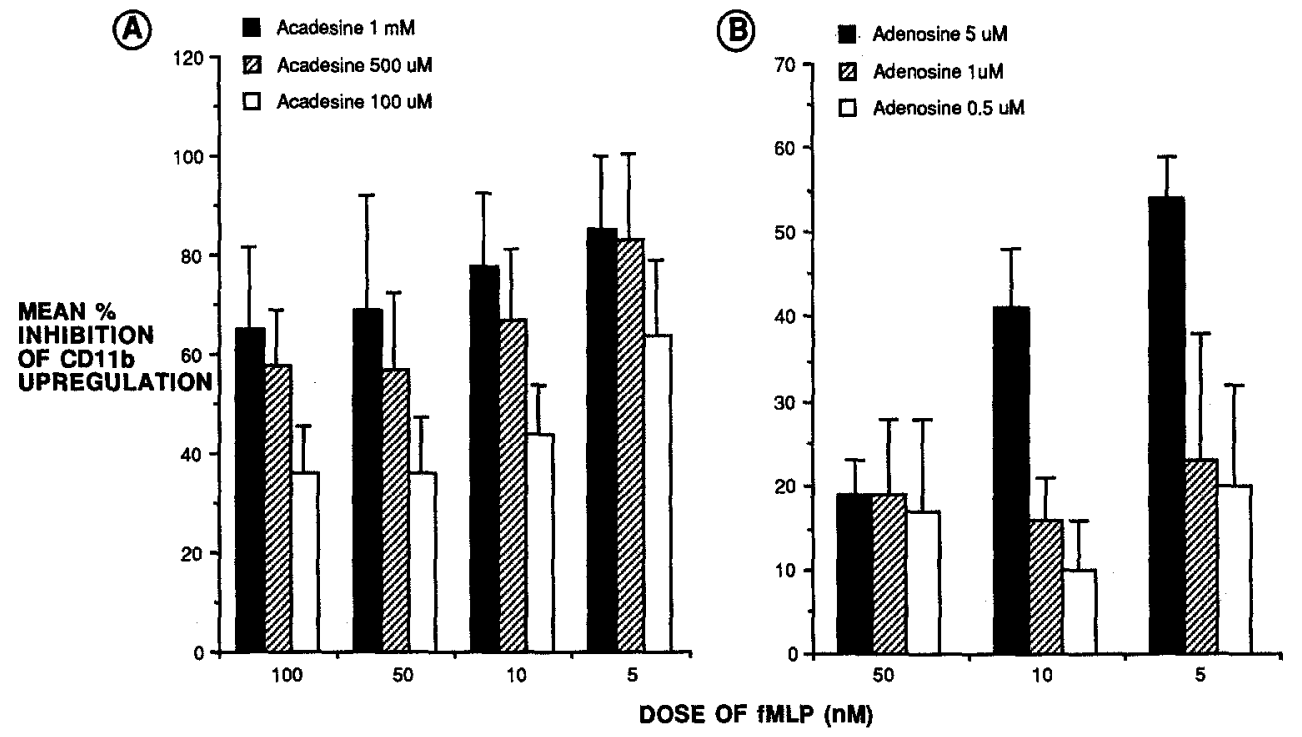

Fig. 1. Percentage inhibition of PMN CD11b up-regulation. Heparinized whole blood was incubated with acadesine (A), adenosine (B), or control diluent, followed by stimulation with fMLP (15 minutes) at the doses shown on the $\mathrm{x}$-axis. Samples were labeled with monoclonal antibodies to CD11b as described in the Materials and methods section and mean CD11b fluorescence on PMN was measured. All CD11b fluorescence values were normalized to control CD11b (diluent incubation followed by fMLP). Values shown represent the mean $\pm S E M$ for three experiments. Both acadesine and adenosine significantly inhibited CD11b up-regulation in response to fMLP $(p<0.01)$, and this effect was dose-related $(p<0.05)$.

CPB, peaking 18 hours after termination of bypass at 3 times the baseline value. CD11b expression on $\mathrm{PMN}$ also rose significantly in the placebo group $(p<0.01)$ to a value 4 times baseline, but, unlike monocytes, PMN CD11b peaked earlier (at termination of $\mathrm{CPB}$ ) and remained elevated 18 hours after CPB at approximately 2.5 times baseline.

When treatment groups were compared with patients receiving placebo for increases in $\mathrm{CD} 11 \mathrm{~b}$ expression using two-factor analysis of variance, there was no difference in the increase in monocyte $\mathrm{CD} 11 \mathrm{~b}$ in the two treatment groups when examined singly as compared with the placebo group. Similarly, the changes in PMN CD11b in the acadesine treatment groups when examined singly did not reach statistical significance $(p=0.16)$. However, when low- and high-dose treatment groups were combined and compared with the placebo group, there was a significant $(p=0.05)$ blunting of the PMN CD11b up-regulation induced by CPB in the acadesine group (Fig. 2, A). PMN CD11b expression in the treatment group peaked at $2.8 \pm 0.3$ (mean $\pm \mathrm{SEM}$ ) times baseline values, as compared with $4.3 \pm 0.9$ (mean \pm SEM) times baseline for the placebo group. Increases in monocyte $\mathrm{CD} 11 \mathrm{~b}$ re- mained unaffected by acadesine treatment when high- and low-dose treatment groups were combined (Fig. 2, $B$ ).

Surface expression of CD11a was similarly examined on PMN and monocytes (Fig. 3) and was found in the placebo group to increase significantly $(p<$ 0.01 ) during CPB only on monocytes. Monocyte CD11a expression increased to $1.5 \pm 0.2$ (mean \pm SEM) times baseline at termination of CPB and increased further to $2.2 \pm 0.3$ (mean \pm SEM) times baseline 18 hours after CPB. However, there was no difference in CD11a up-regulation in monocytes for acadesine groups when analyzed singly versus the placebo group or after combination of the low- and high-dose treatment groups.

The number of circulating PMNs increased slightly during CPB and continued to increase 18 hours after CPB in all patients (Table I). The combined low- and high-dose treatment groups demonstrated a higher PMN count; however, the difference did not reach statistical significance $(p=0.14)$. The absolute number of monocytes was similarly unaffected by acadesine treatment, whether groups were examined singly or low- and high-dose treatment groups were combined. 

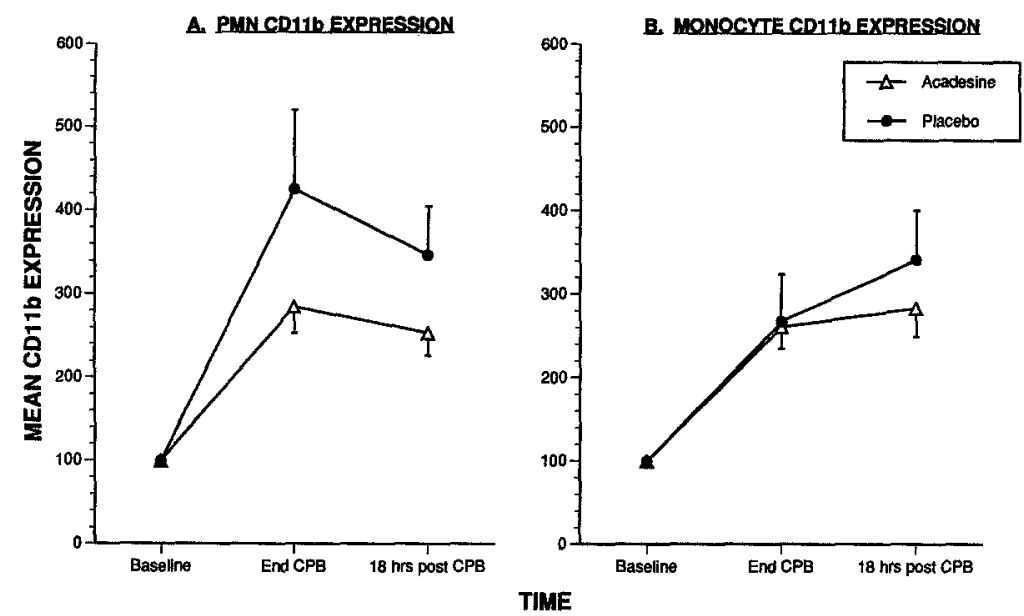

Fig. 2. Leukocyte CD11b on CPB. Total surface CD11b was measured on PMNs (A) and monocytes (B) in whole blood taken before the operation (Baseline), at termination of CPB (End CPB), and 18 hours after $\mathrm{CPB}$ (18 hrs post CPB) and is expressed as a percentage of the baseline fluorescence value. Patients receiving placebo are shown in solid circles $(n=11)$ and patients receiving low- or high-dose acadesine in the open triangles $(n=23)$. Each measurement is the mean \pm SEM. Up-regulation of PMN CD11b was significantly inhibited by acadesine treatment when compared with placebo $(p=0.05)$. Increases in monocyte CD11b were not affected by acadesine treatment $(p=0.55)$.
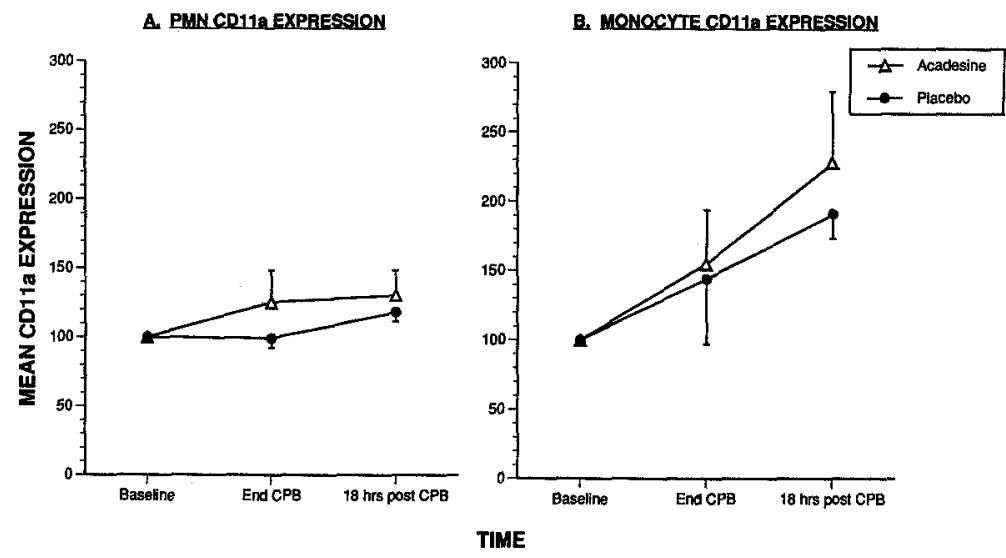

Fig. 3. Leukocyte CD11a on CPB. Total surface CD11a was measured on PMNs (A) and monocytes (B) in whole blood, as described in Fig. 2, and similarly divided into a placebo group (solid circles) and acadesine-treated group (open triangles). Only monocyte CD11a increased significantly $(p=0.01)$ in both the placebo group and acadesine-treated patients, and there was no difference in CD11b expression between these two groups $(p=0.73)$.

Platelet adhesion receptors ( $P$-selectin and GPIb modulation). The percentage of circulating platelets expressing surface $\mathbf{P}$-selectin increased significantly in all patients $(p<0.05)$ by termination of CPB and into the post-CPB period (data not shown), as has been previously reported. ${ }^{13-15}$ Similarly, surface expression of the von Willebrand factor adhesion receptor, GPIb, decreased significantly in all patients $(p<0.01) 18$ hours after CPB to $86 \% \pm 1.7 \%$ (mean \pm SEM) of baseline values. ${ }^{15,16,18}$ The increased in P-selectin expression and the decrease in GPIb did not differ between any of the groups when analyzed singly or when low- and high-dose acadesine groups were combined.

Platelet-leukocyte adhesion. For the 34 patients taken as a single group, the percentage of leukocytes with bound platelets was comparable between leukocyte subsets before the start of the operation, with 
Table I. Mean PMN counts $\pm S E M$

\begin{tabular}{lcccc}
\hline & Baseline & End CPB & 18 hr after CPB & $p$ Value* \\
\hline PMNs $\left(\times 10^{6} / \mathrm{ml}\right)$ & & & & \\
$\quad$ Placebo & $3.91 \pm 0.45$ & $5.69 \pm 1.01$ & $1.94 \pm 1.32$ & 0.14 \\
$\quad$ Low + high & $4.02 \pm 0.34$ & $7.29 \pm 0.93$ & & \\
Monocytes $\left(\times 10^{6} / \mathrm{ml}\right)$ & & & $0.80 \pm 0.17$ & 0.58 \\
$\quad$ Placebo & $0.55 \pm 0.07$ & $0.35 \pm 0.08$ & $0.92 \pm 0.07$ & \\
Low + high & $0.59 \pm 0.05$ & $0.35 \pm 0.06$ & \\
\hline
\end{tabular}

*For placebo versus low + high.

$16 \% \pm 1.9 \%$ (mean \pm SEM) of monocytes and $11 \% \pm 1.3 \%$ of PMNs binding platelets. These values are similar to those previously described. ${ }^{13}$ The percentage of circulating monocyte-platelet conjugates increased significantly in all groups, peaking at the end of CPB with a mean of $40 \% \pm$ $3.3 \%$ (SEM) of circulating monocytes having bound platelets $(p<0.01)$. The percentage of circulating PMN-platelet conjugates also increased significantly in all patients at termination of CPB $(p<0.05)$, but to a much lower percentage $(15 \% \pm 1.6 \%$ SEM) than monocytes. There was no difference in the degree of platelet-leukocyte adhesion for either leukocyte subset in the treatment groups when analyzed either singly or in combination versus the placebo group.

\section{Discussion}

In this investigation we have demonstrated in vitro that both acadesine and adenosine are capable of significantly inhibiting the increase in PMN CD11b induced by the PMN agonist fMLP. This in vitro observation is supported by the in vivo demonstration that acadesine similarly inhibits the upregulation of PMN CD11b during CPB. This effect appears specific for $\mathrm{CD} 11 \mathrm{~b}$ and for granulocytes (PMN CD11a and monocyte CD11a and CD11b were not affected).

CD11a/CD18 and CD11b/CD18 are members of the $\beta_{2}$ integrin family of adhesion receptors, which have a common $\beta$-subunit (CD18) and a distinct $\alpha$-subunit (CD11a, CD11b, CD11c), CD11a/CD18 and CD11b/CD18 in particular permit PMN adhesion to and diapedesis through endothelial cells. ${ }^{8-10}$ PMN CD11b/CD18-mediated adhesion is necessary for hydrogen peroxide secretion in response to the PMN agonists fMLP and platelet-activating factor. $^{11,12}$ In animal models of myocardial and lung injury, antibodies to CD11b have been shown to attenuate the injury associated with infarction and reperfusion ${ }^{33,34}$ or complement infusion. ${ }^{35}$ In human beings, CD11b has been shown to be up- regulated in PMNs and monocytes across the coronary circulation in patients with unstable angina. ${ }^{36}$ Thus CD11b/CD18 up-regulation may result from, as well as contribute to, tissue damage induced by ischemia. By contrast, PMN CD11a/CD18 appears to be more important in the adhesion of resting PMNs to activated endothelial cells. CD11a surface expression is not changed in response to chemotactic stimulation ${ }^{37}$ or, as demonstrated in this study, by PMN activation occurring during CPB.

Adenosine has been shown in vitro to inhibit both fMLP-induced superoxide radical formation by PMNs and PMN adhesion to endothelial cells. ${ }^{38,39}$ These effects have been shown to correlate with a reduction in infarct size in animal models of myocardial infarction and reperfusion injury. ${ }^{40,41}$ Acadesine is a purine nucleoside analog that has been classified as an "adenosine-regulating agent" because of its capacity to increase adenosine accumulation in tissues where ischemia drives cells to net adenosine triphosphate catabolism. ${ }^{1}$ By increasing endogenous adenosine levels locally, acadesine has been shown to improve postischemic contractile function, increase subendocardial blood flow without inducing coronary steal, augment myocardial preconditioning, attenuate myocardial stunning, and decrease granulocyte accumulation in ischemic myocardium. $^{1,3}$ These effects have been shown to correlate with a reduction in infarct size in animal models of myocardial infarction and reperfusion injury. ${ }^{4,6}$

$\mathrm{We}^{13}$ have previously reported that $\mathrm{CPB}$ results in significant up-regulation of CD11b on both PMNs and monocytes in the systemic circulation, and comparable degrees of $\mathrm{CD} 11 \mathrm{~b}$ up-regulation were found in the placebo group in the current study. Patients in the present study were also studied for changes in CD11a, but, as mentioned earlier, only monocytes demonstrated a significant increase in this adhesion receptor. The specific agent(s) responsible for this increase are not known, but candidates include intermediaries in the complement and con- 
tact activation pathways, both of which are activated by CPB. ${ }^{42,43}$ The in vivo arm of this study was conducted on patients at our institution who were enrolled in multicenter phase II and III trials of acadesine. As noted in the preliminary report of phase II of the multicenter study, acadesine produced a $64 \%$ reduction in myocardial infarction (defined by both the appearance of de novo $Q$ waves and an increase in the levels of the myocardial [MB] fraction of creatine kinase) ${ }^{30,31}$ When low and high doses were combined from all institutions, the acadesine-treated patients had a lower incidence $(29 \%)$ of creatine kinase MB spillage than the placebo group $(47 \%)$, with $p=0.05$. The blunting of PMN CD11b up-regulation by in vivo acadesine, which we have demonstrated in our much smaller number of patients, was unexpected in view of the fact that acadesine has been thought to have localized effects under conditions of tissue ischemia resulting in adenosine triphosphate catabolism, but not necessarily systemic effects. However, our in vitro studies demonstrate that in whole blood under normoxic conditions, acadesine is capable of inhibiting the increases in CD11b induced by fMLP. Acadesine would seem to offer significant advantages over adenosine, because in the therapeutic range acadesine is associated with minimal effects on cardiac conduction and vascular tone. ${ }^{44,45} \mathrm{~Pa}$ tients receiving acadesine demonstrated higher total PMN counts on average, perhaps resulting from their lower surface expression of adhesion receptors; however, this difference was not statistically significant in the current study.

Both acadesine and adenosine demonstrated dose-related in vitro inhibition of CD11b upregulation. The dose-responsiveness was seen at doses that varied by fivefold and tenfold. The lack of apparent dose-responsiveness observed in vivo is likely due to the relatively small numbers of patients studied together with the fact that low- and high-dose treatment groups differed by only a factor of 2 . The inhibition of myocardial ischemia measured in phase II of the study was also not dose-related ${ }^{31}$ but phase III demonstrated a reduction in infarction incidence and creatine kinase $\mathrm{MB}$ release in patients having $Q$ waves at the higher dose (D. Mangano and the Multicenter Study of Perioperative Ischemia Research Group, personal communication).

Both adenosine and acadesine have been shown to inhibit platelet aggregation in previous in vitro studies. ${ }^{2,46}$ We hypothesized that this inhibitory effect might result from inhibition of platelet gran- ule release, comparable with the inhibition of CD11b up-regulation from its preformed stores. We measured changes in platelet expression of P-selectin and GPIb, as well as P-selectin-dependent platelet-leukocyte adhesion during CPB, to determine whether acadesine might exert an inhibitory effect on platelet adhesion receptors in vivo. Acadesine did not inhibit P-selectin expression, loss of GPIb, or the increase in P-selectin-dependent platelet-leukocyte conjugate formation induced by $\mathrm{CPB}$. This suggests that the effects of acadesine during CPB are relatively specific for inhibition of PMN activation, as opposed to a more general suppression of cellular activation.

PMNs have been increasingly implicated in the pathophysiology of myocardial infarction and reperfusion injury. ${ }^{47,48}$ Advances in thrombolytic therapy and balloon angioplasty have spurred efforts to inhibit PMN-mediated tissue injury and thereby salvage newly reperfused myocardium. The current study implicates direct inhibition of up-regulation of the adhesion receptor, CD11b, by adenosine and acadesine as one possible mechanism for their ability to prevent PMN-mediated injury in myocardial ischemia.

We thank the Multicenter Study of Perioperative Ischemia Research Group, which conducted the phase II and III acadesine trials.

\section{REFERENCES}

1. Mullane K. Acadesine: the prototype adenosine regulating agent for reducing myocardial ischaemic injury. Cardiovasc Res 1993;27:43-7.

2. Bullough D, Zhang C, Mullane K. Acadesine (AICA riboside) inhibits platelet aggregation in human whole blood [Abstract]. Int J Purine Pyrimidine Res 1991; 2(Suppl 1):66.

3. Mentzer RM Jr, Ely SW, Lasley RD, Berne RM. The acute effects of AICAR on purine nucleotide metabolism and postischemic cardiac function. J THORAC Cardiovasc SuRg 1988;95:286-93.

4. Galiñanes M, Bullough D, Mullane K, Hearse D. Sustained protection by acadesine against ischemiaand reperfusion-induced injury. Circulation 1992;86: 589-97.

5. Levin R, Eberle M, Marchetta P, Cronstein B. Stimulation of adenosine release from human endothelial cells inhibits neutrophil function [Abstract]. Circulation 1990;82:111-77.

6. Gruber H, Hoffer M, McAllister D, et al. Increased adenosine concentration in blood from ischemic myocardium by AICA riboside: effects on flow, granulocytes, and injury. Circulation 1989;80:1400-11. 
7. Bullough D, Fox M, Potter S, Metzner K, Young M, Mullane K. AICA riboside protects myocardial tissue from reperfusion injury and oxidant induced damage [Abstract]. FASEB J 1991;5:A1436.

8. Albelda S, Buck C. Integrins and other cell adhesion molecules. FASEB J 1990;4:2868-80.

9. Smith C, Marlin S, Rothlein R, Toman C, Anderson D. Cooperative interactions of LFA-1 and Mac-1 with intercellular adhesion molecule-1 in facilitating adherence and transendothelial migration of human neutrophils in vitro. J Clin Invest 1989;83:2008-17.

10. Arnaout M. Structure and function of the leukocyte adhesion molecules CD11/CD18. Blood 1990;75:103750.

11. Shappell S, Toman C, Anderson D, Taylor A, Entman M, Smith C. Mac-1 (CD11b/CD18) mediates adherence-dependent hydrogen peroxide production by human and canine neutrophils. J Immunol 1990;144: 2702-11.

12. Nathan C, Srimal S, Farber C, et al. Cytokine induced respiratory burst of human neutrophils: dependence on extracellular matrix proteins and CD11/CD18 integrins. J Cell Biol 1989;109:1341-9.

13. Rinder C, Bonan J, Rinder H, Mathew J, Hines R, Smith B. Cardiopulmonary bypass induces leukocyteplatelet adhesion. Blood 1992;79:1201-5.

14. Rinder C, Bonnert J, Rinder H, Mitchell J, Ault K, Hillman R. Platelet activation and aggregation during cardiopulmonary bypass. Anesthesiology 1991;74:38893.

15. Rinder C, Mathew J, Rinder H, Bonan J, Ault K, Smith B. Modulation of platelet surface adhesion receptors during cardiopulmonary bypass. Anesthesiology 1991;75:563-70.

16. George J, Pickett E, Saucerman S, et al. Platelet surface glycoproteins: studies on resting and activated platelets and platelet microparticles in normal subjects and observations in patients during adult respiratory distress syndrome and cardiac surgery. J Clin Invest 1986;78:340-8.

17. Wenger RK, Ludasiewicz H, Mikuta BS, Niewiarowski S, Edmunds HJ. Loss of platelet fibrinogen receptors during clinical cardiopulmonary bypass. J THOrac Cardiovasc Surg 1989;97:235-9.

18. van Oeveren W, Hander MP, Roozendaal KJ, Eijsman L, Wildevuu CRH. Aprotinin protects platelets against the initial effect of cardiopulmonary bypass. J Thorac Cardiovasc Surg 1990;99:788-97.

19. Kondo C, Tanaka K, Takagi K, et al. Platelet dysfunction during cardiopulmonary bypass surgery with special reference to platelet membrane glycoproteins. ASAIO J 1993;39:M550-3.

20. Carmody M, Ault KA, Mitchell JG, Rote NS, Ng A. Production of monoclonal antibodies specific for platelet activation antigens and their use in evaluating platelet function. Hybridoma 1990;9:631-5.
21. McGregor J, Brochier J, Wild F, et al. Monoclonal antibodies against platelet membrane glycoproteins. Eur J Biochem 1983;131:427-30.

22. Ruan C, Du X, Xi X, Castaldi P, Berndt M. A murine glycoprotein $\mathrm{Ib}$ complex monoclonal antibody SZ2 inhibits platelet aggregation induced by ristocetin and collagen. Blood 1987;69:570-4.

23. Beverley P. Production and use of monoclonal antibodies in transplantation immunology. In: Touraine JL, Trager J, Betuel J, et al, eds. Transplantation and clinical immunology XI. Amsterdam: Excerpta Medica, 1980:87-93.

24. Ross G, Cain J, Lachmann P. Membrane complement receptor type three $\left(\mathrm{CR}_{3}\right)$ has lectin-like properties analogous to bovine conglutinin and functions as a receptor for zymosan and rabbit erythrocytes as well as a receptor for iC3b. J Immunol 1985;134:3307-11.

25. Rinder H, Bonan J, Rinder C, Ault K, Smith B. Dynamics of leukocyte-platelet adhesion in whole blood. Blood 1991;78:1730-7.

26. Shattil S, Cunningham M, Hoxie J. Detection of activated platelets in whole blood using activationdependent monoclonal antibodies and flow cytometry. Blood 1987;70:307-17.

27. Rinder H, Murphy M, Mitchell J, Stocks J, Ault K, Hillman R. Progressive platelet activation with storage: evidence for shortened survival of activated platelets after transfusion. Transfusion 1991;31:40914.

28. Kuijpers T, Tool A, van der Schoot C, et al. Membrane surface antigen expression on neutrophils: a reappraisal of the use of surface markers for neutrophil activation. Blood 1991;78:1105-11.

29. Stewart AG, Harris T. Adenosine inhibits plateletactivating factor, but not tumor necrosis factor- $\alpha-$ induced priming of human neutrophils. Immunology 1993;78:152-8.

30. Leung J, Stanley T, Mathew J, et al. Effects of acadesine on perioperative cardiac morbidity in a placebo controlled, double blind study [Abstract]. J Am Coll Cardiol 1992;19:112A.

31. Leung J, Stanley T, Mathew J, et al. An initial multicenter, randomized controlled trial on the safety and efficacy of acadesine in patients undergoing CABG surgery. Anesth Analg 1994;78:420-34.

32. Ault $\mathrm{K}$, Rinder $\mathrm{H}$, Mitchell J, Rinder C, Lambrew $\mathrm{C}$, Hillman R. Correlated measurement of platelet release and aggregation in whole blood. Cytometry 1989;10:448-55.

33. Simpson P, Todd R III, Fantone J, Michelson J, Griffin J, Lucchesi B. Reduction of experimental canine myocardial reperfusion injury by a monoclonal antibody (anti-Mol, anti-CD11b) that inhibits leukocyte adhesion. J Clin Invest 1988;81:624-8.

34. Todd R III, Simpson P, Lucchesi B. Anti-inflammatory properties of monoclonal anti-Mol (CD11b/ 
CD18) antibodies in vitro and in vivo. In: Springer TA, Anderson DC, Rosenthal A, Rothlein R, eds. Leukocyte adhesion molecules. New York: SpringerVerlag, 1988:125-37.

35. Mulligan M, Smith C, Anderson D, et al. Role of leukocyte adhesion molecules in complement-induced lung injury. J Immunol 1993;150:2401-6.

36. Mazzone A, De Servi S, Ricevuti G, et al. Increased expression of neutrophil and monocyte adhesion molecules in unstable coronary artery disease. Circulation 1993;88:358-63.

37. Springer TA. Adhesion receptors of the immune system. Nature 1990;346:425-34.

38. Burkey T, Webster R. Adenosine inhibits fMLPstimulated adherence and superoxide anion generation by human neutrophils at an early step in signal transduction. Biochem Biophys Acta 1993;1175: 312-8.

39. Cronstein B, Levin R, Belnoff J, Weissman G, Hirschhorn R. Adenosine: an endogenous inhibitor of neutrophil-mediated injury to endothelial cells. $\mathrm{J}$ Clin Invest 1986;78:760-70.

40. Olafsson B, Forman M, Puett D, et al. Reduction of reperfusion injury in the canine preparation by intracoronary adenosine: importance of the endothelium and the no-reflow phenomenon. Circulation 1987;76: 1135-45.
41. Ely S, Berne R. Protective effects of adenosine in myocardial ischemia. Circulation 1992;85:893-904.

42. Wachtfogel YT, Harpel PC, Edmunds LH Jr, Colman $\mathrm{RW}$. Formation of $\mathrm{Cl}_{\mathrm{S}} \mathrm{Cl}$-inhibitor, kallikrenin-C1-inhibitor, and plasmin- $\alpha_{2}$-plasmin inhibitor complexes during cardiopulmonary bypass. Blood 1989;73:468-74.

43. Kirklin JK, Westaby S, Blackstone EH, Kirklin JW, Chenoweth DE, Pacifico AD. Complement and the damaging effects of cardiopulmonary bypass. J THORAC CARDIOVASC Surg 1983;86:845-51.

44. Dixon R, Gouris J, McDermott D, Fujitaki J, Dewland P, Gruber H. AICA-riboside: safety, tolerance, and pharmacokinetics of a novel adenosine-regulating agent. J Clin Pharmacol 1991;31:342-7.

45. Ogilby JD, Jaekyeong $H$, Iskandrian A. Effect of adenosine on coronary blood flow and its use as a diagnostic test for coronary artery disease. Cardiovasc Res 1993;27:48-53.

46. Kitakaze M, Hori M, Sato H, Takashima S, Kitabatake A. Endogenous adenosine inhibits formation of microthromboembolism in ischemic myocardium [Abstract]. Circulation 1990;82(Suppl):III276.

47. Forman M, Virmani R, Puett D. Mechanisms and therapy of myocardial reperfusion injury. Circulation 1990;81(Suppl):IV69-78.

48. Entman M, Michael L, Rossen R, et al. Inflammation in the course of early myocardial ischemia. FASEB J 1991;5:2529-37.

\section{1-800-55-MOSBY}

This number links you to the full text of articles published in over 25,000 journals, including all Mosby Journals. MOSBY Document Express ${ }^{\mathrm{TM}}$, a rapid response information retrieval service, provides quick turnaround, 24-hour availability, and speedy delivery methods. For inquiries and pricing information, call our toll-free, 24-hour order line: 1-800-55-MOSBY; outside the United States: 415-259-5046; fax: 415-259-4019; E-mail: mosbyexp@class.org. 